\title{
Predictions on Preserving Bone Mass in Knee Arthroplasty With Bisphosphonates
}

\author{
Jeffry S. Nyman, PhD, ${ }^{*}$ Juan J. Rodrigo, MD, $\dagger$ Scott J. Hazelwood, PhD,* \\ Oscar C. Yeh, PhD,* and R. Bruce Martin, PhD*
}

\begin{abstract}
Using a computational model of bone adaptation, we investigated the long-term ability of bisphosphonates to minimize proximal bone loss that is associated with stress shielding in the tibia after long-stemmed total knee arthroplasty (TKA). When invoking bisphosphonate effects, the remodeling activity was suppressed, and the resorption size was reduced. Compared with the untreated simulation, bisphosphonate slowed the rate of bone loss after TKA (42\% reduction in bone loss at 1 year). Activating the drug 3 months before the surgery reversed bone loss associated with the reduction in such activities as walking, but it did not provide any substantial benefit in the long-term. Late bisphosphonate treatment did not reverse the bone loss that occurred 3.5 years after TKA, although it preserved $3 \%$ of bone normally lost without treatment. Key words: stress shielding, TKA, bisphosphonate, finite element analysis, press fit.
\end{abstract}

Implant failure in total joint arthroplasty may be prevented when adequate fixation of the implant components is achieved after surgery and when this fixation is maintained over the long-term [1]. Although mechanically stiff stems with a large diameter and extensive porous coating (which facilitates bony ingrowth) provide the initial stability and fixation, these attributes, when applied to femoral prostheses in total hip arthroplasty (THA), have been found to be associated with more pronounced periprosthetic bone resorption [2]. Moreover, this bone loss compromises long- term fixation, and loosening of the prosthesis may occur [3].

Bone loss after total joint arthroplasty occurs, in part, because the relatively stiffer implants reduce the physiological loading of the contiguous bone, leading to disuse bone remodeling. The majority of such bone loss typically occurs within the first year after total joint arthroplasty and has been observed by serial x-ray absorptiometry in the proximal femur after THA [4-8]. Similar findings have been observed after total knee arthroplasty (TKA) in the distal femur [9-11] as well as in the proximal tibia [12-15]. Through implant design (eg, peg or stem), material selection (eg, cobalt chrome alloy or titanium alloy), and fixation technique (eg, press fit or cement), attempts have been made to minimize this phenomenon. Although such efforts have improved the longevity of total joint arthroplasty, mechanical-related bone loss (ie, stress shielding) has not been prevented, and multiple revision surgeries are still an eventuality, especially for young patients (younger than 50 years) with a reconstructed joint who are 
typically more active and live longer than older patients with a joint arthroplasty.

It is well documented that the drug alendronate increases bone mineral density (BMD) in the femoral neck and trochanter of postmenopausal women with osteoporosis [16-25]. This bisphosphonate drug may be equally effective in preventing or reversing bone loss associated with stress shielding. There is the possibility, however, that bisphosphonates may disrupt the important process of bone apposition to implant surfaces, which ensures adequate fixation, because bisphosphonates can inhibit mineralization (especially at high dosages). Allaying such a concern, Frenkel et al [26] and Mochida et al [27] found that alendronate did not inhibit bony integration to various titanium surfaces and hydroxyapatite-coated femoral stems, respectively, in a dog model.

Whether bisphosphonates can prevent bone loss associated with total joint arthroplasty as they do for postmenopausal bone has been explored in both animals and human beings. Besides stress shielding, bone loss may result from the cellular response (ie, macrophages) to wear debris. Therefore, using a rat model of particulate-induced osteolysis, alendronate was studied and found to prevent or reverse bone loss in the tibia [28] and the femur [29] when polyethylene particles were injected near the implant. Because there was more bone around the implant in the group subjected to particulates and alendronate, although osteoclasts were present, than in the nontreated group (implant only), Millett et al [28] concluded that alendronate reduces osteoclastic activity, thereby reducing bone resorption and preserving bone.

Venesmaa et al [30] and Soininvaara et al [31] investigated whether alendronate could prevent periprosthetic bone loss in the human femur after THA and TKA, respectively. Each prospective randomized trial found that the alendronatetreated group had a significantly smaller decrease in BMD than the calcium only-treated group after 6 months to 1 year of total joint arthroplasty. Similarly, Wilkinson et al [32] found that a single dose of another bisphosphonate drug, pamidronate, significantly reduced proximal bone loss in the femur by 26 weeks after THA compared with a control. Moreover, the drug was not associated with more adverse events (eg, cardiac failure and diarrhea) than normally occur after THA without bisphosphonate treatment.

Although these studies suggest that bisphosphonates can reduce bone loss after total joint arthroplasty, the question still remains whether this effect can be maintained over the long term. To investi- gate this in a relatively short time frame, bisphosphonate effects were simulated here with existing computational models of bone remodeling developed for the tibia with long-stemmed TKA [33] and for bisphosphonate treatment [34]. Additional questions that were investigated with this theoretical model were (1) would bisphosphonates given 3 months before surgery provide better protection against bone loss than those given immediately after surgery? (2) do bisphosphonates recover bone that has already been lost to stress shielding? and (3) what are the relative contributions of suppression of remodeling activity and reduction in resorption area to minimizing bone loss?

\section{Materials and Methods}

\section{Bone Adaptation Model}

Changes in bone mass after long-stemmed TKA with and without bisphosphonate treatment were simulated using a finite element model of the tibia coupled with a theoretical model of bone remodeling [33]. In the theoretical model, which is described by Hazelwood et al [35], bone loss associated with disuse was assumed to occur when a mechanical stimulus $(\Phi)$ dropped below a disuse threshold $\left(\Phi_{0}\right)$. In each element of bone, the mechanical stimulus was estimated from the principal strains $(\varepsilon)$ of 3 daily loading activities and their respective loading frequency $\left(R_{\mathrm{L}}\right)$ :

$\Phi=\sum_{i=1}^{3} \varepsilon^{4} R_{L i}$

When the mechanical stimulus is less than the disuse threshold, the activation frequency (Ac.f) of basic multicellular units (BMUs), the teams of cells that remove and then replenish packets of bone, were increased in both cortical and trabecular bone. The disuse-related increase in remodeling activity resulted in bone loss caused by an increase in the number of resorption sites. Also, if the porosity was greater than $20 \%$ and the bone was in a state of disuse, bone formation decreased relative to resorption in each BMU, thereby causing negative bone turnover or disuse remodeling:

Formation $=0.5 \times$ Resorption $\left(1+\frac{\Phi}{\Phi_{0}}\right)$

In other words, the bone balance ratio (formation per resorption) at the BMU level was less than 1 when bone was in state of disuse. The model tracked changes in the amount of bone occupying a representative area within each element via the history of activation frequency as BMUs progress 


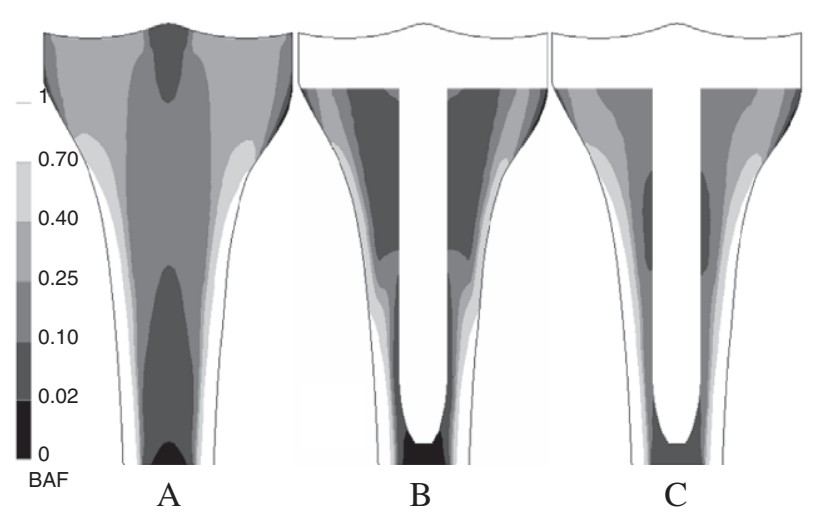

Fig. 1. Comparing distribution of BAF in the nonoperated tibial bone at steady state (A) to that for TKA at 700 days postoperative TKA reveals a greater loss of bone when bisphosphonates are not simulated (B) than when their effects are included immediately postoperative (C).

through the resorption, reversal, and formation periods. Bone mass was thus calculated as bone area per total area or bone area fraction (BAF).

\section{Bone Remodeling Simulations}

Starting with a homogeneous material state throughout the tibia and applying physiological joint reaction forces, the bone mass distribution was allowed to evolve until each variable of the model reached a steady state as described in a previous study [33]. Briefly, the joint reaction force was evenly distributed normal to each condyle surface in 1 situation, and then the other situations had medial-biased $(70 \% / 30 \%)$ or lateral-biased $(30 \% / 70 \%)$ loading with a $5^{\circ}$ tilt to account for lateral or medial ground reaction forces. The model calculated tibial surface strains in the physiological range, as measured by Burr et al [36]. Once a steady state was achieved, the tibia model exhibited a normal anatomic distribution of cortical and cancellous bone that was used as the starting point for the current study (Fig. 1A).
From the equilibrium, normal condition, the forces and loading frequencies acting on the model were reduced by $10 \%$ and $22 \%$, respectively, to simulate loss of loading activity caused by osteoarthritic pain [37]. Bone remodeling was then allowed to evolve for another 200 days. We did not include any aging or disease effects on the material behavior of bone (ie, the relationship between BAF and modulus did not change).

A long-stem version of TKA was selected so that the effectiveness of bisphosphonate in preserving bone mass would be investigated in a severe case of stress shielding. We set the TKA components, comprised of a $16 \times 120 \mathrm{~mm}$ press fit, titanium alloy stem with a cemented tibial tray, and ultrahigh molecular weight polyethylene insert, to their respective material properties (Table 1) and ran implant simulations for the succeeding 6.5 years without bisphosphonate treatment or with treatment starting on either the day of TKA, 3 months before TKA, or 3.5 years after TKA. Joint reaction forces, but not loading frequency, were returned to normal after 100 days. Bone loss was quantified below the tibial tray as percent change from the intact tibial BAF after reduction in loading activity.

\section{Bisphosphonate Effects}

The 2 main antiresorptive actions of bisphosphonate are the inhibition of BMU activation frequency and reduction in erosion by the BMU [40]. Because these actions occur when the bisphosphonate comes in contact with an osteoclast and suppression is greatest in regions of high remodeling activity [41], the potency of suppressing activation frequency was a function of the number of resorbing BMUs.

$P=P_{\max }\left(1-e^{-\tau_{s} \times N \cdot R s . B M U}\right)$

where $P_{\max }$ is the maximum suppression coefficient, $\tau_{\mathrm{s}}$ is the rate of suppression coefficient, and N.Rs.BMU is the number of resorbing BMUs. Bisphosphonate treatment was simulated in the

Table 1. Material Properties for the Components of TKA and for Bone

\begin{tabular}{lcccc}
\hline Material & Young's modulus (MPa) & Poisson's ratio & Location & Reference \\
\hline PMMA & 2150 & 0.46 & Below tray & Lewis et al [38] \\
UHMWPE & 2300 & 0.25 & Tibial insert & Lewis et al [38] \\
Titanium & 79000 & 0.36 & Tray and stem & Lewis et al [38] \\
Cortical & $23400(\mathrm{BAF})^{5.74}$ & 0.30 & BAF >0.8; Fig. 2A & Turner et al [39] \\
Trabecular & $14300(\mathrm{BAF})^{1.33}$ & 0.30 & BAF $\leq 0.8 ;$ Fig. 2A & Turner et al [39] \\
\hline
\end{tabular}

Abbreviation: UHMWPE, ultrahigh molecular weight polyethylene. 
model by suppressing BMU activation frequency by the percentage of $P$, and then independent of activation frequency suppression, the normal resorption by BMUs was reduced by 3/13th, corresponding to the reduction in erosion depth observed by Chavassieux et al [42]. In a previous study by Nyman et al [34], these antiresorptive actions were invoked in a representative volume of postmenopausal trabecular bone. With a maximum suppression coefficient of 1.0, a rate of suppression coefficient of 5 , and a reduction in the erosion depth by 3/13th, the simulation predicted an increase in bone mass similar to that observed by dual-energy x-ray absorptiometry over 7 years in the spines of postmenopausal women who were treated daily with $10 \mathrm{mg}$ of alendronate [24]. More specifically, the simulation matched the clinically observed $4 \%$ increase in BMD over the first 6 months of treatment. It also matched the decreasing rate of BMD increase between 6 months and 7 years of treatment. Therefore, these coefficients were invoked when simulating bisphosphonate effects in the TKA model.

In 2 additional simulations of bisphosphonate treatment starting on the day of TKA, (a) the rate of suppression coefficient was increased to 20 without changing the reduction in resorption and (b) resorption was reduced by $2 / 7$ th without changing the rate of suppression. The former action effectively increases the suppression of activation frequency of new BMUs, whereas the latter further reduces the size of resorption space created by BMUs. This sensitivity analysis was done to investigate the contributions of activation suppression and bone balance, respectively, on inhibiting bone loss associated with stress shielding (ie, mechanically induced disuse bone remodeling). To elucidate the underlying mechanism of bone loss in the model, mechanical stimulus, activation frequency, and bone balance were recorded (as an average within the region of interest) over time for each simulation.

\section{Results}

Compared with the untreated simulation, bisphosphonate treatment preserved bone mass after TKA (Fig. 2). Furthermore, it prevented the thinning of the cortices that can occur with TKA. Its antiresorptive effects, however, did not completely prevent proximal bone loss associated with stress shielding but slowed the progression of loss (Fig. 2). One year after TKA, there was an average of $42.0 \%$ less bone loss below the tibial tray with bisphosph-

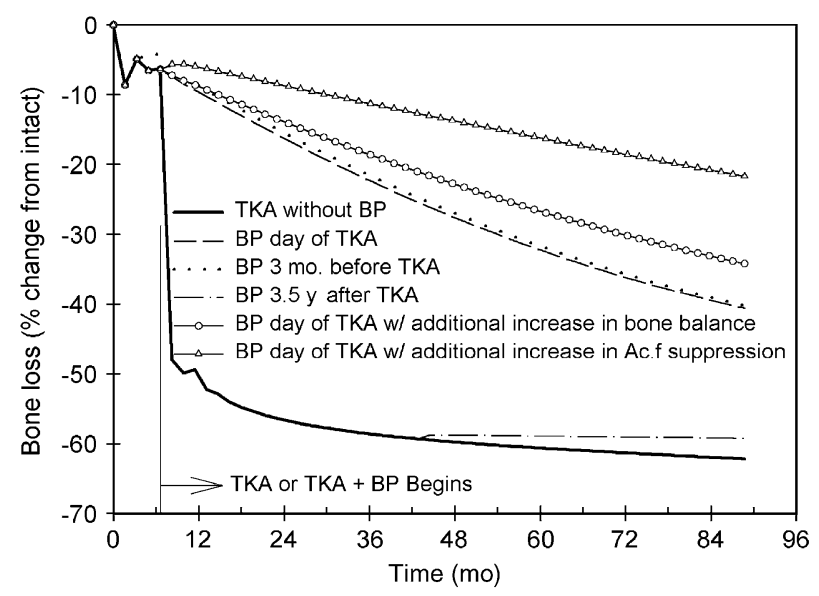

Fig. 2. The rate of bone loss after TKA below the tibial tray is slower when bisphosphonate effects are simulated than without treatment. Abbreviation: BP, bisphosphonate.

onate treatment given the day of surgery than without bisphosphonate treatment. Activating the drug 3 months before TKA reversed some of the bone loss associated with the reduction in loading activity that typically occurs with osteoarthritic knees, but it did not provide any substantial benefit in the long term. Bisphosphonate given 3.5 years after TKA did not reverse the bone loss that occurred up to that time after surgery, although it slowed the rate of loss (saved 3\% of bone normally lost at 6.5 years). Increasing activation frequency suppression potency of a bisphosphonate drug had a greater effect than reducing the resorption size (ie, improving the bone balance) in preserving bone over the long term.

Decreasing the loading activity lowered the mechanical stimulus below the disuse threshold and subsequently caused bone loss (Fig. 2). Reducing bone resorption by invoking bisphosphonate treatment before TKA caused positive bone turnover within the BMU (ie, more bone was added than removed as shown in Fig. 3), and bone loss was temporarily reversed (dotted line in Fig. 2). When the long-stemmed TKA was simulated at 6 months after reduction in loading, bone loss in the model resumed, despite the pharmacological suppression of bone resorption and the return of normal joint reaction forces. In the model of long-stem TKA without drug intervention, significant stress shielding had 2 consequences: (a) increased remodeling activity introduced porosity and (b) bone formation in each BMU decreased relative to resorption. This elevated rate of remodeling subsided over time as the reduction of bone 


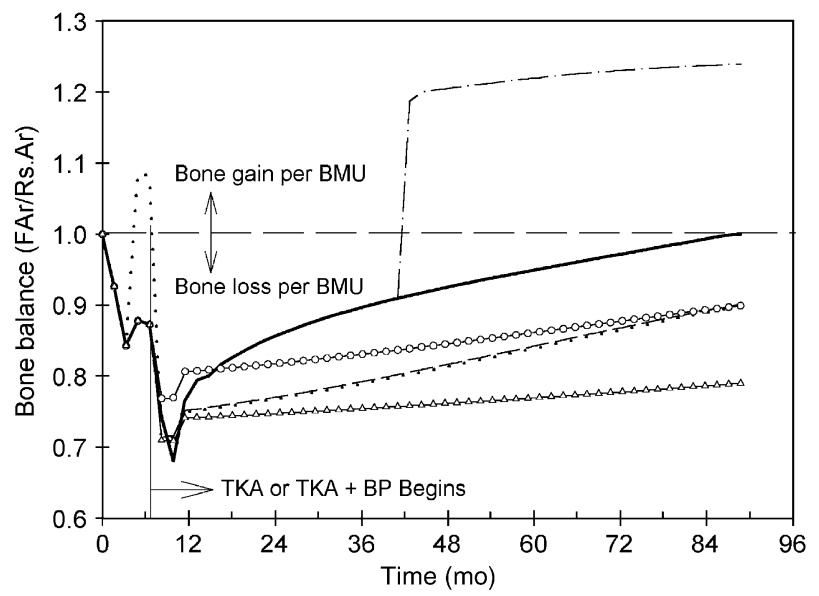

Fig. 3. Bone balance, the ratio of formation area (FAr) to resorption area (Rs.Ar) is less than 1 because the state of disuse caused by the TKA overrides the increase provided by bisphosphonate unless the drug is given 3.5 years postoperatively.

mass increased the strain. With bisphosphonate treatment, the stimulation of disuse remodeling by TKA was suppressed. However, there was still a decrease in bone formation, and although the drug decreased BMU erosion depth, formation remained less than resorption (Fig. 3). In other words, the drug did not overcome the consequences of stress shielding but delayed them.

\section{Discussion}

The objective of simulating the antiresorptive action of bisphosphonate in a hypothetical bone adaptation model of long-stem TKA was to investigate (1) whether the drug minimizes bone loss associated with stress shielding over the long term, (2) whether giving the drug in advance of surgery provided any additional benefit, and (3) whether the drug could recover bone mass that had already been lost because of stress shielding. Secondary objectives included investigating the relative contributions of activation frequency suppression and resorption size reduction in minimizing bone loss and understanding the underlying mechanisms of bone loss.

Bisphosphonate treatment in the present study minimized bone loss after TKA, reducing bone loss by almost one half below the tibial tray during the 6.5 -year period. Bone loss was not completely stopped because (a) remodeling was suppressed but not completely blocked and (b) a disuse state or stress shielding was prolonged compared with
TKA without treatment. To clarify, without antiresorptive action, bone strains increased after their initial decrease as bone stiffness was lost to stress shielding. Subsequently, the mechanical stimulus returned to the threshold level, and the bone balance approached one (Fig. 3) as the rate of bone loss slowed. With bisphosphonate treatment, however, the preservation of bone kept the strain on bone at a reduced level. Therefore, despite the reduction in bone resorption relative to normal bone formation, the disuse state imposed a negative bone turnover (ie, resorption was greater than formation).

Despite an improvement in the bone balance ratio (ie, increased positive bone turnover per BMU) when bisphosphonate treatment was given 3.5 years after TKA, there was no significant gain in bone. This was because of the rather low remodeling activity occurring at the time of treatment. There were too few BMUs to cause perceptible increase in bone mass. For a gain to occur, a pharmacological agent would likely need to promote bone formation in addition to suppressing bone resorption.

In the present model, invoking a more sensitive suppression of remodeling activity (a rate of suppression coefficient of 20 instead of 5) caused fewer number of resorbing BMUs, whereas invoking a greater reduction in resorption by osteoclasts (71.4\% of normal formation instead of $76.9 \%$ of normal formation) caused a more positive bone turnover per BMU. Interestingly, the model predicted that increasing the potency of a bisphosphonate drug further reduces the amount of bone formed at the BMU level (compare the dotted line to the triangle line in Fig. 3) but still reduces the amount of bone loss overall. This was because of the fact that there were fewer BMUs to remove bone with high suppression. Based on the predictions of the present bone remodeling simulations, greater potency of remodeling suppression should be a higher priority than greater potency of resorption reduction when designing bisphosphonate drugs for TKA.

To the best of our knowledge, the effect of bisphosphonates on tibial bone density after TKA has not been studied. Moreover, there are no clinical studies that have measured bone loss below the tibial tray for long-stemmed TKA. Nonetheless, there are 3 clinical observations that support the suitability of the present analytical model. First, several studies observed that the majority of bone loss in the tibia after TKA occurs in the first year and diminishes thereafter $[12,14,15,43]$. This trend was predicted by the model when simulating TKA 
without bisphosphonate treatment (Fig. 2). Second, the predicted thinning of the cortices by the model (Fig. 1B) has been observed clinically after TKA by Seitz et al [44]. Third, Karbowski et al [12] reported that a tibial endoprosthesis with stem length of $40 \mathrm{~mm}$ caused an average $9.26 \%$ decrease in tibial bone density below the tray after 9 months of TKA, and Regner et al [15] reported a tibial endoprosthesis with stem length of $60 \mathrm{~mm}$ that caused an average $22 \%$ decrease in tibial bone density in a similar region. From a mechanical viewpoint, bone loss increases with an increase in stem length [45]. Thus, a greater bone loss than found clinically for shorter stems would occur for a $120-\mathrm{mm}$ stem as the model predicts. Lastly, bone loss did occur in the short term after THA or TKA when patients were given bisphosphonates [30-32], and it was significantly less than in patients who were not treated with the drug. The model also predicted much less bone loss for treated TKA compared with TKAs without the actions of bisphosphonate.

The present findings come from a hypothetical model of TKA bone adaptation, so they should be interpreted in light of the limitations of the model. Such limitations included those related to 2-dimensional finite element models: plane strain assumptions, exclusion of out-of-plane forces, simplification of geometry, and idealization of material behavior. Nonetheless, the model predicts the morphological characteristics of the tibia (cortices at the diaphysis, medullary canal, and cancellous bone in metaphysis) given the few hypothetical loading activities. One limitation in the simulation of bisphosphonate treatment is the absence of the contribution of secondary mineralization to bone mass. Because bisphosphonates slow remodeling activity, there is more time for bone to mineralize, which increases mass, before being replaced with fresh osteoid [46]. This is an additive effect suggesting that the present study overpredicts bone loss with the drug. Another possible limitation is the independence between the fixed reduction in resorption size and suppression of remodeling activity. Unlike activation frequency suppression, resorption reduction was not a function of the number of resorbing BMUs (ie, as the presence of osteoclasts increased, the resorbing efficiency or BMU life span did not decrease proportionally). The consequence of this is unclear because resorption size affects both porosity (hence, stiffness of bone) and microdamage removal (hence, the demand for activation frequency).

The results of the present study suggest that bisphosphonate treatment starting on the day of
TKA may slow the progression of bone loss associated with stress shielding. Preserving bone mass over the long term would reduce the likelihood of aseptic loosening, a common cause for revision. There appears to be no advantage of giving the drug in advance to prevent bone loss because of a reduction in loading activity, and starting the administration of the drug several years after surgery may only provide modest benefits. Because of known side effects and a long half-life (>10 years), routine use of bisphosphonate in TKA would require a long-term clinical study.

\section{Acknowledgments}

The authors thank the ARCS foundation, Inc, for providing monetary assistance to J.S.N. Support for S.J.H. was provided by the Whitaker Foundation grant RG-02-0630 and a UC Davis Health System Research Award. NIH grants AR41644 and AR42844 supported the initial development of the theoretical model of bone remodeling used in this study.

\section{References}

1. Bauer TW, Schils J. The pathology of total joint arthroplasty. II. Mechanisms of implant failure. Skeletal Radiol 1999;28:483.

2. Bobyn JD, Mortimer ES, Glassman AH, et al. Producing and avoiding stress shielding. Laboratory and clinical observations of noncemented total hip arthroplasty. Clin Orthop 1992;274:79.

3. Taylor M, Tanner KE. Fatigue failure of cancellous bone: a possible cause of implant migration and loosening. J Bone Joint Surg Br 1997;79:181.

4. Aldinger PR, Sabo D, Pritsch M, et al. Pattern of periprosthetic bone remodeling around stable uncemented tapered hip stems: a prospective 84-month follow-up study and a median 156-month crosssectional study with DXA. Calcif Tissue Int 2003; 73:115.

5. Karrholm J, Anderber C, Snorrason F, et al. Evaluation of a femoral stem with reduced stiffness. A randomized study with use of radiostereometry and bone densitometry. J Bone Joint Surg Am 2002; 84:1651.

6. Kroger H, Venesmaa P, Jurvelin J, et al. Bone density at the proximal femur after total hip arthroplasty. Clin Orthop 1998;352:66.

7. Marchetti ME, Steinberg GG, Greene JM, et al. A prospective study of proximal femur bone mass following cemented and uncemented hip arthroplasty. J Bone Miner Res 1996;11:1033.

8. Venesmaa PK, Kroger HP, Miettinen HJ, et al. Monitoring of periprosthetic BMD after uncemented 
total hip arthroplasty with dual-energy x-ray absorptiometry - a 3-year follow-up study. J Bone Miner Res 2001;16:1056.

9. Bryan JM, Sumner DR, Hurwitz DE, et al. Altered load history affects periprosthetic bone loss following cementless total hip arthroplasty. J Orthop Res 1996; 14:762.

10. Petersen MM, Olsen C, Lauritzen JB, et al. Changes in bone mineral density of the distal femur following uncemented total knee arthroplasty. J Arthroplasty 1995; 10:7.

11. van Loon CJ, Oyen WJ, de Waal Malefijt MC, et al. Distal femoral bone mineral density after total knee arthroplasty: a comparison with general bone mineral density. Arch Orthop Trauma Surg 2001; $121: 282$.

12. Karbowski A, Schwitalle M, Eckardt A, et al. Periprosthetic bone remodelling after total knee arthroplasty: early assessment by dual energy $x$-ray absorptiometry. Arch Orthop Trauma Surg 1999; 119:324.

13. Lonner JH, Klotz M, Levitz C, et al. Changes in bone density after cemented total knee arthroplasty: influence of stem design. J Arthroplasty 2001; 16:107.

14. Petersen MM, Nielsen PT, Lauritzen JB, et al. Changes in bone mineral density of the proximal tibia after uncemented total knee arthroplasty. A 3-year follow-up of 25 knees. Acta Orthop Scand 1995;66:513.

15. Regner LR, Carlsson LV, Karrholm JN, et al. Bone mineral and migratory patterns in uncemented total knee arthroplasties: a randomized 5-year follow-up study of 38 knees. Acta Orthop Scand 1999;70:603.

16. Black DM, Cummings SR, Karpf DB, et al. Randomised trial of effect of alendronate on risk of fracture in women with existing vertebral fractures. Fracture Intervention Trial Research Group. Lancet 1996; 348:1535.

17. Bone HG, Downs Jr RW, Tucci JR, et al. Doseresponse relationships for alendronate treatment in osteoporotic elderly women. Alendronate Elderly Osteoporosis Study Centers. J Clin Endocrinol Metab 1997;82:265.

18. Bone HG, Greenspan SL, McKeever C, et al. Alendronate and estrogen effects in postmenopausal women with low bone mineral density. Alendronate/ Estrogen Study Group. J Clin Endocrinol Metab 2000;85:720.

19. Chesnut III CH, McClung MR, Ensrud KE, et al. Alendronate treatment of the postmenopausal osteoporotic woman: effect of multiple dosages on bone mass and bone remodeling. Am J Med 1995; 99:144.

20. Devogelaer JP, Broll H, Correa-Rotter R, et al. Oral alendronate induces progressive increases in bone mass of the spine, hip, and total body over 3 years in postmenopausal women with osteoporosis. Bone 1996;18:141.
21. McClung M, Clemmesen B, Daifotis A, et al. Alendronate prevents postmenopausal bone loss in women without osteoporosis. A double-blind, randomized, controlled trial. Alendronate Osteoporosis Prevention Study Group. Ann Intern Med 1998; 128:253.

22. Pols HA, Felsenberg D, Hanley DA, et al. Multinational, placebo-controlled, randomized trial of the effects of alendronate on bone density and fracture risk in postmenopausal women with low bone mass: results of the FOSIT study. Fosamax International Trial Study Group. Osteoporos Int 1999; 9:461.

23. Stepan JJ, Vokrouhlicka J. Comparison of biochemical markers of bone remodelling in the assessment of the effects of alendronate on bone in postmenopausal osteoporosis. Clin Chim Acta 1999;288:121.

24. Tonino RP, Meunier PJ, Emkey R, et al. Skeletal benefits of alendronate: 7-year treatment of postmenopausal osteoporotic women. Phase III Osteoporosis Treatment Study Group. J Clin Endocrinol Metab 2000;85:3109.

25. Bone HG, Hosking D, Devogelaer JP, et al. Ten years' experience with alendronate for osteoporosis in postmenopausal women. N Engl J Med 2004; 350:1189.

26. Frenkel SR, Jaffe WL, Valle CD, et al. The effect of alendronate (Fosamax) and implant surface on bone integration and remodeling in a canine model. J Biomed Mater Res 2001;58:645.

27. Mochida Y, Bauer TW, Akisue T, et al. Alendronate does not inhibit early bone apposition to hydroxyapatite-coated total joint implants: a preliminary study. J Bone Joint Surg Am 2002;84:226.

28. Millett PJ, Allen MJ, Bostrom MP. Effects of alendronate on particle-induced osteolysis in a rat model. J Bone Joint Surg Am 2002;84:236.

29. Thadani PJ, Waxman B, Sladek E, et al. Inhibition of particulate debris-induced osteolysis by alendronate in a rat model. Orthopedics 2002;25:59.

30. Venesmaa PK, Kroger HP, Miettinen HJ, et al. Alendronate reduces periprosthetic bone loss after uncemented primary total hip arthroplasty: a prospective randomized study. J Bone Miner Res 2001; $16: 2126$

31. Soininvaara TA, Jurvelin JS, Miettinen HJ, et al. Effect of alendronate on periprosthetic bone loss after total knee arthroplasty: a one-year, randomized, controlled trial of 19 patients. Calcif Tissue Int 2002; 71:472.

32. Wilkinson JM, Stockley I, Peel NF, et al. Effect of pamidronate in preventing local bone loss after total hip arthroplasty: a randomized, double-blind, controlled trial. J Bone Miner Res 2001;16:556.

33. Nyman JS, Hazelwood SJ, Rodrigo JJ, et al. Long stemmed total knee arthroplasty with interlocking screws: a computational bone adaptation study. J Orthop Res 2004;22:51.

34. Nyman JS, Yeh OC, Hazelwood SJ, et al. A theoretical analysis of long-term bisphosphonate effects on 
trabecular bone volume and microdamage. Bone 2004;35:296.

35. Hazelwood SJ, Martin RB, Rashid MM, et al. A mechanistic model for internal bone remodeling exhibits different dynamic responses in disuse and overload. J Biomech 2001;34:299.

36. Burr DB, Milgrom C, Fyhrie D, et al. In vivo measurement of human tibial strains during vigorous activity. Bone 1996;18:405.

37. Robon MJ, Perell KL, Fang M, et al. The relationship between ankle plantar flexor muscle moments and knee compressive forces in subjects with and without pain. Clin Biomech (Bristol, Avon) 2000; 15:522.

38. Lewis G, Jonathan V, Kambhampati S. Effect of material property representation on stresses in endoprostheses. Biomed Mater Eng 1998;8:11.

39. Turner $\mathrm{CH}$, Anne V, Pidaparti RM. A uniform strain criterion for trabecular bone adaptation: do continuum-level strain gradients drive adaptation? J Biomech 1997;30:555.

40. Fleisch H. Bisphosphonates: mechanisms of action. Endocr Rev 1998;19:80.
41. Lin JH. Bisphosphonates: a review of their pharmacokinetic properties. Bone 1996;18:75.

42. Chavassieux PM, Arlot ME, Reda C, et al. Histomorphometric assessment of the long-term effects of alendronate on bone quality and remodeling in patients with osteoporosis. J Clin Invest 1997; 100:1475.

43. Bohr HH, Lund B. Bone mineral density of the proximal tibia following uncemented arthroplasty. J Arthroplasty 1987;2:309.

44. Seitz P, Ruegsegger P, Gschwend N, et al. Changes in local bone density after knee arthroplasty: the use of quantitative computed tomography. J Bone Joint Surg Br 1987;69:407.

45. Askew MJ, Lewis JL. Analysis of model variables and fixation post length effects on stresses around a prosthesis in the proximal tibia. J Biomech Eng 1981;103:239.

46. Hernandez CJ, Beaupre GS, Marcus R, et al. A theoretical analysis of the contributions of remodeling space, mineralization, and bone balance to changes in bone mineral density during alendronate treatment. Bone 2001;29:511. 